\title{
Where did I park my car? Influence of landmark permanency on navigation
}

\author{
Charlotte Roy1, Dennis Wiebusch1 and Marc Ernst1 \\ 1 Ulm University, Ulm 89081, Germany
}

\begin{abstract}
.
Visual landmarks provide crucial information for human navigation. But what defines a landmark? To be uniquely recognized, a landmark should be distinctive and salient, while providing precise and accurate positional information. It should also be permanent, e.g., to find back your car, a nearby church seems a better landmark compared with a truck, as you learned the truck might likely move. To this end, we here investigate the learning of landmark permanency while treating permanency as a probabilistic characteristic for human navigation. Particularly we study the learning behaviour when exposed to landmarks whose permanency feature is probabilistically defined. We hypothesize that humans will be able to learn this feature and assign higher weight to more permanent landmarks. To test the hypothesis, we used a homing task where participants had to return to a position that was surrounded by three landmarks. In the learning phase we manipulated the permanency of one landmark by secretly repositioning it prior to returning to the home position. The statistics of repositioning was drawn from a normal distribution. In the test phase we investigated the weight allocated to the nonpermanent landmark by analysing its influence on the navigational performance. The first experiment revealed the probabilistic nature of learning the prior of landmark's permanency, the second experiment confirmed the results in modifying the statistic of the prior. The third and the fourth experiments showed that priors of permanency can be updated by experiences highlighting the capacity of adaptation to the environment.
\end{abstract}

Keywords: Human navigation, Landmark, Permanency. 


\section{Introduction}

"Humans and other mobile animals move about their environments in order to get to places with food, mates, shelters, margaritas and other resources" (p.257, Montello, 2005). This definition explains simply why we navigate every day and how crucial this ability is for us. In order to navigate through the environment -to get to places- animals, including humans, can use different strategies: beaconing, path-integration, route-following, map-compass and cognitive map (Geva-Sagiv et al., 2015). The combination of these strategies or the switch from one to another enables effective navigation (Etchamendy \& Bohbot, 2007; Igloi, Zaoui, Berthoz, \& Rondi-Reig, 2009). However, successful navigation seems to imply predominantly visual information, which is an important factor in the beaconing strategy, also named landmark-based strategy (Foo et al., 2005; Knierim et al., 1998). Therefore, improving our understanding of landmark-based navigation is important, as far as an impairment of navigation ability with age has been linked with the capacity of landmark coding (Bécu et al., 2019). In order to move from A to B, humans and animals can use external sensory cues, like a visual object, a sound or an odor. Out of the multitudes of objects surrounding us in the world, we use only a fraction to navigate. This brings up the question: what makes an object a landmark? This question has been of interest for many years leading to a collection of properties like size, shape, color, intensity, orientation, topology, visibility, uniqueness, brevity, etc. (Burnett, 2000; Caduff \& Timpf, 2008; Sorrows \& Hirtle, 1999). Among them, permanency, or stability, seems an important feature for successful navigation (Biegler \& Morris, 1996; Burgess et al., 2004). For example, to find back your car, a nearby church tends to be a better landmark compared to a truck, because you learned that the truck likely might move. The ability to assess the feature of permanency of an object seems to be related to the more general ability to navigate. Auger and colleagues reported a link between the ability to discriminate the permanency of the landmarks and the general navigation skill, implying the retrosplenial cortex. The better participants estimated the permanency of an object, the better navigators they were (Auger et al., 2012, 2017; Auger \& Maguire, 2013). Permanency of a landmark is a crucial feature to move through the environment. It is therefore important to understand how we learn this feature.

Within the Bayesian Decision Theory framework, we hypothesize that humans learn the permanency of an object in a probabilistic way. The theory predicts that a decision is based on the combination of priors and sensory information, where the weight allocated to one or the other depends on their reliabilities, assessed by the variance of their distributions; the higher the variance, the lower the allocated weight (Ernst \& Bülthoff, 2004; Maloney \& Mamassian, 
2009). While the theory has been mainly applied to model perception, here we apply the mechanisms to navigational behavior (see also Cheng, Shettleworth, Huttenlocher, \& Rieser, 2007; Kiryakova, Aston, Beierholm, \& Nardini, 2019; Nardini, Jones, Bedford, \& Braddick, 2008). The probability that we have experienced a church moving is much lower than that of experiencing a moving truck. This knowledge is called a prior. The priors that we have acquired during our lifetime have direct consequences on our behavior (Adams et al., 2004; Parise et al., 2014). In the present experiment, we manipulate the priors of the participants by training them in two different conditions: permanent vs non-permanent. We expect an effect of the priors on the navigational behavior. Therefore, we investigate the behavior of participants when exposed to landmarks whose permanency is probabilistically defined.

Our main hypothesis is that humans learn the feature of landmark's permanency in a probabilistic way. Depending on the trained prior, the same object can be alternatively used as permanent or non-permanent. Compared to a permanent object, a non-permanent object is assumed to be less reliable and thus weighted less. Consequently, it is expected to have less influence on the navigational performance.

For all the experiments we used a simple form of navigation: a homing task, in which participants have to go from A to B and then back home from B to A (Etienne \& Jeffery, 2004). The home position was surrounded by three objects that participants could use as landmarks. All experiments started with a training phase, followed by a test phase. For all experiments we compare two conditions, permanent versus non-permanent. The test phases of both conditions were similar, while the training phases differed. In the permanent training all objects remained at the same place during each training trial. However, in the non-permanent training, we manipulated the permanency of one object by secretly repositioning it before the participant turned around to return home. We assumed that non-permanency is learned by the repetitive experience of an object whose position changes. In the test phases, we evaluated the weight allocated to the objects by analyzing its influence on the navigational performance.

The first experiment revealed the probabilistic nature of learning the feature of landmark's permanency. The second experiment confirmed the result in modifying the statistics of the object. The third and the fourth experiments showed that priors are adaptable and can be modified according to the requirements of the environment.

\section{Experiment 1}


Here, we evaluate the effect of landmark's permanency on navigational performance, including the homing performance and the path travelled. Navigational performance is assessed by the ability to go back home as well as to locate home. The first ability is assessed by the trajectory of the path travelled by the participants. The second, called homing performance, is measured by the distance between the response of the participants and the correct home position. Participants performed a homing-task under two different conditions, permanent versus nonpermanent. We expect an effect of the trained prior on the navigational behavior.

\section{Method}

Participants. 15 participants (9 male, 6 female) aged between 19 and $28(m=22.2, s d=3.1)$ took part in the first experiment. The sample size was determined based on an effect size from a similar experiment (Cohen's $d z=0.98$; Jetzschke, Ernst, Froehlich, \& Boeddeker, 2017), aiming for a power of 0.95 with alpha $=0.05$ (two-tailed). All participants gave their written informed consent before participating and received course credits or money $(7 € / \mathrm{h})$. The experiment was approved by the ethics committee of Ulm University.

\section{Material \& Procedure.}

For the task the 8-monitor OctaVis virtual reality set-up was used (Dyck et al., 2012). The monitors are Elo TouchSystems ET2639L 26-inch touch screens that are installed in portrait orientation and form an octagonal shape with a diameter of 0.96 meters (see Figure 1). With 7.75 degrees covered by the screen frames between two display surfaces, the display area covered about 298 degrees in the horizontal plane. The screens' display mode was set to 1366x768@60Hz, resulting in a pixel size of 0.4215 x 0.4215mm.

Participants were seated on a rotatable chair in the center of the set-up, using a Microsoft Xbox wireless game controller in order to navigate in the virtual environment. While the analog stick of the controller was used to perform translational movements, rotation of the chair allowed for changes in orientation. Combining translation and rotation, the participants were able to move into the direction in which the chair was facing. Further interaction in the virtual environment was possible by performing touch gestures on the screens and using buttons of the controller. A custom-built OpenGL renderer that maintained a refresh rate of $60 \mathrm{~Hz}$ was utilized to visualize the virtual environment. The visualization was configured such that one unit of length in the virtual environment corresponded to one meter in the real world. Each participant's trajectory in the virtual environment was recorded at a frequency of $5 \mathrm{~Hz}$. Finally, the rotation 
of the chair was recorded each time an update was transmitted the built-in controller, which has a resolution of 0.35 degrees.

Different techniques were applied to prevent participants from using their orientation inside the setup (e.g., by memorizing the position of virtual objects on the screens or by moving backwards in a straight line) to cheat while performing the experiment: first, the speed of lateral movement (taking a step to the side) and backwards movement with respect to the orientation of the chair was reduced by a factor of 0.1 , making longer movements in those directions unnerving. Moreover, the orientation of the virtual environment with respect to the orientation of the screens changed by a random amount at the beginning of each trial. This way, the objects' position on the screens (when viewed from the home position) changed on a trial-by-trial basis. Since participants could only rotate themselves but not the virtual environment relative to the screens, they were not able to undo this rotation. Finally, any modification of the non-permanent landmark's position was only performed if the chair was facing at least 135 degrees away from the home position and the participant was at least 12.5 meters away. If these two conditions were not fulfilled at any time the non-permanent landmark was relocated once the participants reached the oasis. The virtual scene was an endless desert, through which participants could freely navigate. The sky was colored monochrome blue, offering no directional cues.

[Figure 1 here]

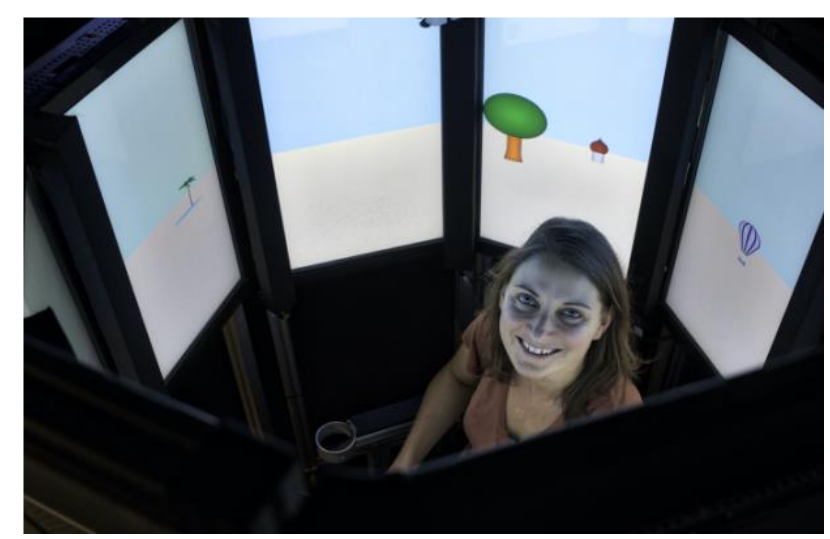

Figure 1. Picture of the experimental setup.

The homing-task consisted in first going to the home position A, then going to the goal B, and afterwards going back to A. The home position was represented by an empty bucket surrounded by three objects: a house, a tree and a hot-air balloon. These objects were chosen for their permanency properties. A tree and a house are rated as very permanent, contrary to the balloon 
which was rated as non-permanent (Auger et al., 2012). Moreover, these three objects were chosen for their similar size and shape in the real world. The three objects were designed to be circular in order to avoid any directional cues. They were positioned in an equilateral triangle with the home-position being located in the center of the triangle, resulting in each object being positioned in a distance of $10 \mathrm{~m}$ from the home-position. Figure 2 shows the experimental design. The goal was represented by an oasis, placed $40 \mathrm{~m}$ from the home position and relocated randomly from trial to trial.

For each trial, participants started from a random location at $20 \mathrm{~m}$ from the home position. Participants were instructed to navigate to the bucket (the home position), to pick it up using the game controller, then go to the oasis (the goal), fill the bucket with water using the game controller once again. As soon as the bucket was filled, the oasis disappeared. Then the task was to go back to the home position and place the bucket at its original position. To select that position, participants had to touch one of the screens with their dominant index finger. Feedback on their performance was provided only in the learning trials. It was given in the form of a colored plant that grew at the home position as well as by awarding points. To receive a point, participants had to place the bucket within an area of 2 meters around the actual home-position. If rewarded a point, the growing plant was of green color. Otherwise it had a brown, withered look. During the test-trials, no feedback was provided.

In the training trials of the permanent condition, the hot-air balloon was fixed, remaining at the same position from trial to trial. In contrast, in the non-permanent condition, the balloon was secretly repositioned while the participants were on their way to the oasis. The statistics of the repositioning of the balloon was drawn from a normal distribution with a 9 m radius around its usual position (see Figure 2, experiment 1).

In order to quantify the influence of the training trials on the performance, we evaluated the effect of relocation on the results of the testing trials. The relocation of a landmark along one axis shifts the homing result into the direction of the relocated landmark, known as the relocation effect (Jetzschke et al., 2017). Four different relocation distances (10, 12, 14 and 16 meters) were tested in both conditions. For an illustration of the experimental design see Figure 2. In the testing trials, the balloon was relocated along the $y$-axis, which is perpendicular to the line between the other two landmarks and runs through the home position. The balloon was relocated for different distances, resulting in four relocations: for the $0 \mathrm{~m}$ relocation, the balloon was $10 \mathrm{~m}$ away from the home position along the $y$-axis, for the $12 \mathrm{~m}$ relocation, it was at $2 \mathrm{~m}$ away from home, and so on. A crucial point with this manipulation is that none of the participants were aware of the relocation. In accordance with the relocation effect, we expect 
the homing results to be shifted toward the relocated landmark. We expect this effect when participants have learned that the three landmarks are permanent. In contrast, we expect a mitigated effect of relocation when participants have learned that one of the objects is not permanent. We particularly expect this, because the relocated object is the one that also has been learned to be non-permanent, i.e. the balloon. Consequently, participants are expected to not rely on the balloon, since they learned that it is non-permanent and thus their responses should be attracted less, or not at all, by the relocated landmark.

[Figure 2 here]

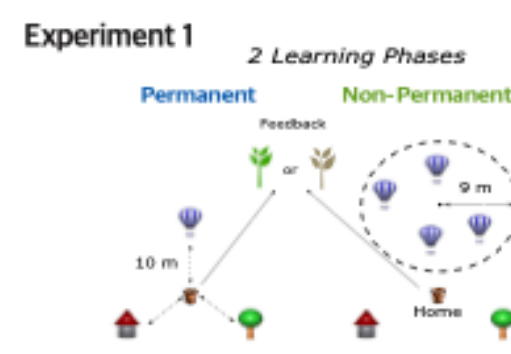

Experiment 2
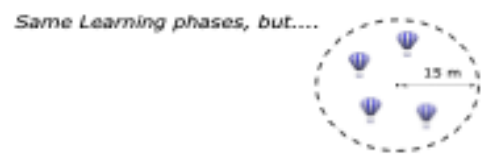

Experiment 3

Same as in Expeirment 2 but... Non-Permanent FIRST
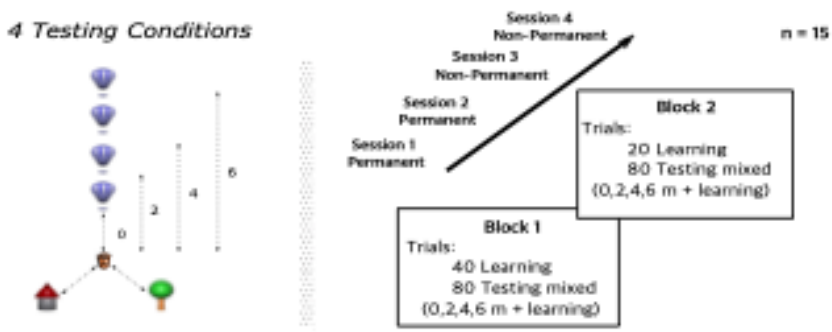

1 Testing Condition
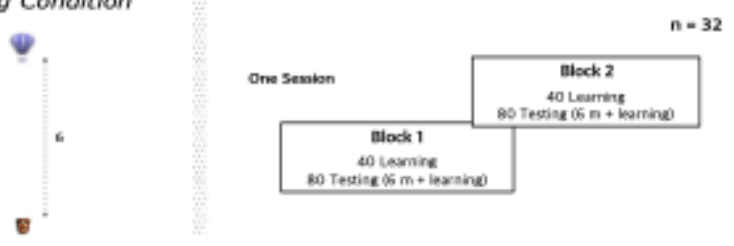

Experiment 4

Permanent

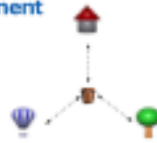

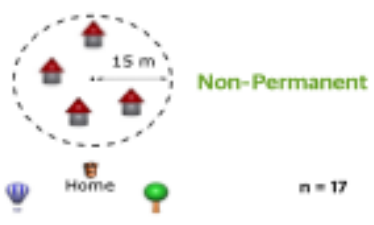

Figure 2. Illustration of the experimental design for the 4 experiments. Experiment 1 includes 4 relocation testing-conditions. Experiment 2 radius of balloon repositionning $=15 \mathrm{~m}$. Experiment 3 , non-permanent condition first. Experiment 4 , balloon replaced by the house.

The experiment was divided in 4 different sessions on 4 different days. All participants performed the entire experiment. The first sessions covered the permanent condition, while the two last sessions addressed the non-permanent condition (see Figure 2). Note that the effect of the conditions' order is tested in experiment 3. Each session, i.e. each day, was divided in 2 blocks. Block 1 started with 40 training trials followed by 80 mixed trials. After that the 
participants took a break of 7 minutes. Then block 2 began with only 20 training trials followed again by 80 mixed trials. Both times the 80 mixed trials consisted of 40 relocations ( 4 distances x 10 trials) and 40 training trials, presented in a random order. For each condition 440 trials were performed: 280 training trials and 160 testing trials $(2$ sessions x 2 blocks x 4 relocations x 10 trials). In total, each participant performed 880 trials ( $440 \times 2$ conditions). The experiment lasted about 10 hours divided in four parts of $2 \mathrm{~h} 30$ each. On average one block lasted 45-55 minutes, while the rest of the time was used to provide instructions and breaks.

Analysis. All the homing performances, training and testing trials, were calculated on the $y$ axis (the axis of the relocation).

A total of three variables were computed. The main variable is the accuracy of the homing performance. It corresponds to the difference between the measured responses of the participant and the correct home location value. The second variable is the precision corresponding to the variance of the distribution of the measurements (Pfuhl et al., 2011). The raw data of the homing performance is shown in Figure 3. We additionally calculated an index measuring the trajectory of the path travelled, named trajectory's index (TI). The variable measures how much the trajectory used by the participant deviated from the ideal straight line towards the home position. The cosine between this line and the direction in which the participant had travelled was calculated for each time frame. For each trial, the mean of all the values was used for the analysis. In order to make high values correspond to the participants moving home in a straight line, the value was inverted (i.e. the difference between 1 and the mean value was calculated) and used as TI. Hence, a value of 1 corresponds to the direct path towards the home position, while 0 would correspond the participants constantly heading in the opposite direction (since they eventually had to reach the home position to finish the trial the mean value cannot be 0 ). Consequently, the higher the TI is, the straighter the path.

We also computed the weight allocated to the relocated landmark. This computation is another way to quantify the relocation effect on the accuracy of the homing performances. Along with our hypothesis, we expect a higher weight for the permanent conditions than for the nonpermanent ones. To calculate the weight, we first calculate the center of the triangle formed by the three objects. Each relocation (except for $0 \mathrm{~m}$ ) leads to new configurations and therefore new triangles and new centers. If the participants relied only on the geometry of the landmarks in terms of distances, i.e. home is positioned in the center of the triangle, then they should place home always in the center of the triangle. Accordingly, we computed the shift of the triangles' center along the $y$-axis for each relocation distance of the balloon: $0 \mathrm{~m}$ is equal to $0 \mathrm{~m}, 2 \mathrm{~m}$ to 
$1.29 \mathrm{~m} ; 4 \mathrm{~m}$ to $2.52 \mathrm{~m}$ and $6 \mathrm{~m}$ to $3.71 \mathrm{~m}$. We divided these values by the homing performances, resulting in weighting the influence of the triangles' center on the performances.

We additionally studied the influence of balloon's position on the homing performances by calculating the correlation between balloon's positions and homing accuracy. The analysis concerns only the learning trails from the non-permanent conditions, corresponding to the trials where the balloon was repositioned. The correlation allows us to investigate more precisely to what extend the balloon's position influence participants' responses.

[Figure 3 here]
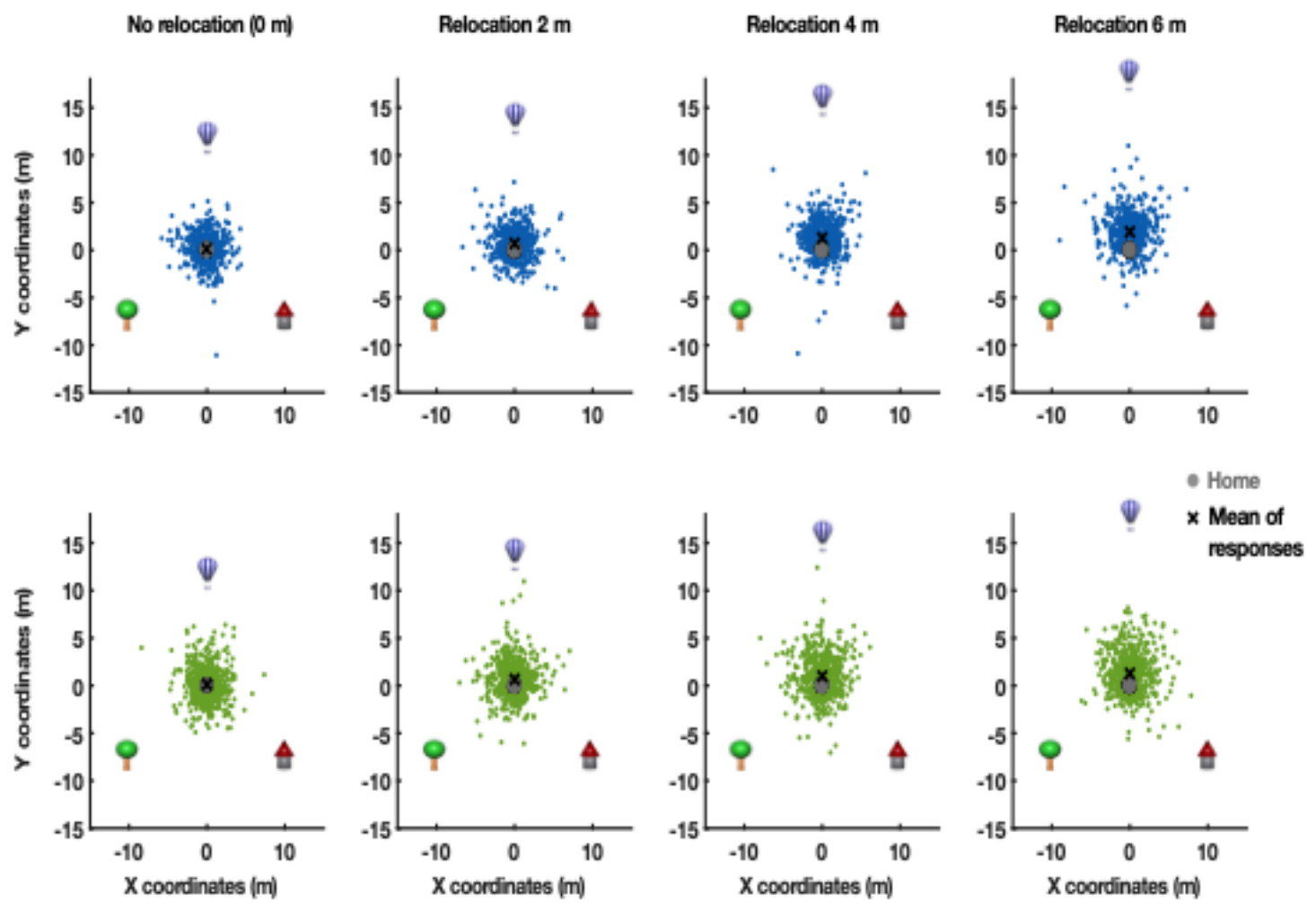

Figure 3. Results from Experiment 1. Each grey dot is the home position, each black cross represents the mean of the responses of the participants. The blue color stands for the permanent conditions of learning, the green color for the non-permanent conditions (balloon secretly respositionned from trial to trial).

Results. Out of the 16 tested participants, one felt the effect of motion sickness preventing him/her to complete the entire experiment. Thus, all analysis is run on 15 participants.

Homing Accuracy. As shown in Figure 4, the relocation effect is influenced by the training of landmark permanency. This pattern is confirmed by a $2 \times 4$ analysis of variance (ANOVA) performed on the mean accuracy of homing responses, with Permanency (permanent, non- 
permanent) and Relocation (0m, 2m, 4m, 6m) as within-subjects factors. There was a main effect of Permanency, $F(1,119)=6.56, p=.017, \eta_{2} \mathrm{p}=0.018$, with higher value of accuracy for permanent than for non-permanent $(1.01 \mathrm{~m}$ vs $0.75 \mathrm{~m})$. Note that for the current study accuracy is not a synonym of good or poor performances but instead a high value of accuracy is a sign of the relocation effect (see also Jetzschke et al., 2017). Therefore, the relocated landmark influenced more the permanent than the non-permanent conditions. There was also a main effect of Relocation, $F(1,119)=38.74, p<.0001, \eta_{2} \mathrm{p}=0.33$, with a linear increase. The further away the relocated landmark was, the higher was the accuracy $(0 \mathrm{~m}$ relocation $=0.14 \mathrm{~m}$, vs 2 $\mathrm{m}$ relocation $=0.68 \mathrm{~m}$, vs $1 \mathrm{~m}$ relocation $=1.14 \mathrm{~m}$, vs $6 \mathrm{~m}$ relocation $=1.59 \mathrm{~m})$. This is another sign of the relocation effect. There was no interaction effect $F(1,119)=2.09, p=.11$.

The weight allocated to the relocated landmark confirms that the relocation effect is influenced by the prior of permanency. The weights corresponded to the triangles' center divided by the homing accuracy. As shown in Figure 4, the performances of all the permanent conditions correspond closely to a weight of 0.5 . Precisely, for $2 \mathrm{~m}$ relocation the weight equaled 0.54 (0.6981/1.29), for $4 \mathrm{~m}$ equaled $0.51(1.2845 / 2.52)$ and for $6 \mathrm{~m}$ the weight corresponded to 0.52 (1.9193/3.71). In the non-permanent conditions, For the shortest relocation, $2 \mathrm{~m}$, the weight equaled $0.51(0.66 / 1.29)$. However, for larger distances the weights decreased. The weight allocated for the $4 \mathrm{~m}$ relocation equaled $0.39(0.9890 / 2.52)$ and for the $6 \mathrm{~m}$ relocation equaled $0.34(1.2681 / 3.71)$. The weight allocated to the landmark configuration is inversely related to the distance of the relocated landmark when the balloon's prior is non-permanent.

Finally, the correlation between balloon's positions and homing accuracy results in a positive correlation, $\sigma 2=0.46, p<.001$. This positive correlation indicates that participants' responses are influenced by the position of the non-permanent landmark.

Homing Precision. There is no significant effect of the relocation effect but a significant effect of the training on the precision of the homing responses (Figure 4). This pattern is confirmed by a 2 x 4 analysis of variance (ANOVA) performed on mean precision of homing responses, with Permanency (permanent, non-permanent) and Relocation (0m, 2m, 4m, 6m) as withinsubjects factors. There was a main effect of Permanency, $F(1,119)=5.81, p=.017, \eta^{2}=0.02$, with a lower value of precision for permanent than for non-permanent (2.67 vs 3.69). A low value of precision indicates more constant performances, participants being able to reproduce their responses more uniformly. Inversely, high values of precision indicate that the distribution is scattered, participants being variable in their responses (see Jetzschke et al., 2017; Pfuhl et 
al., 2011). Therefore, the participants were more constant in their responses for the permanent conditions than for the non-permanent conditions. There were nor main effect of Relocation, $F(1,119)=1.72, p=.17$, neither interaction effect $F(1,119)=0.01, p=.99$.

Trajectory's index. There is no significant effect of the relocation effect but a significant effect of the training on the participants' paths (Figure 4). This pattern is confirmed by a $2 \mathrm{x} 4$ analysis of variance (ANOVA) performed on the trajectory's index, with Permanency (permanent, nonpermanent) and Relocation $(0 \mathrm{~m}, 2 \mathrm{~m}, 4 \mathrm{~m}, 6 \mathrm{~m})$ as within-subjects factors. There was a main effect of Permanency, $F(1,119)=3.98, p=.04, \eta_{2 p}=0.01$, with lower value for permanent than for non-permanent $(0.65$ vs 0.67$)$. The higher the value of the trajectory's index was, the straighter to the home position the paths of the participants were. There was neither a main effect of Relocation, $F(1,119)=0.06, p=.98$, nor an interaction effect $F(1,119)=0.07, p=.97$.

[Figure 4 here]
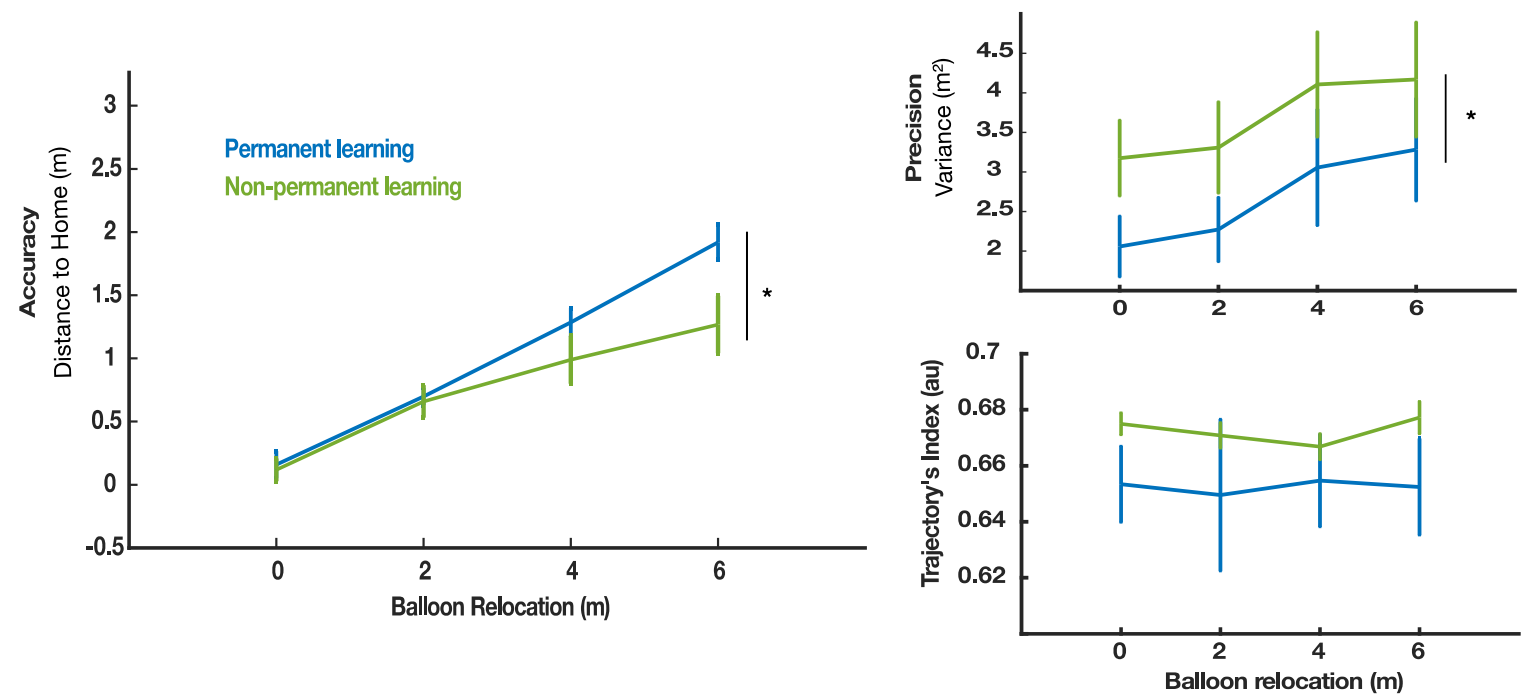

Figure 4. Results from Experiment 1. Accuracy, precision and goodness of trajectory for each relocation condition. Both conditions of learning permanent versus non-permanent. Errors bars indicate SE of the mean. Stars indicate significant differences between both conditions (permanent vs. non-permanent).

\section{Discussion.}


The first experiment shows an effect of the prior of permanency on the navigational performances. The results showed a larger relocation effect for the permanent condition than for the non-permanent condition. Therefore, participants' ability to locate home is related with the permanency of the objects in the surrounding. When an object is permanent, participants are more prone to use it as a landmark, which is less the case when this same object has been experienced as non-permanent. We can conclude then, that an object which is moving is not a reliable landmark. More interesting is that the nature of learning is driven by probability, the repetitive experience of the participants. The same object, here the hot-air balloon, is used differently by the participants according to the previous experience of the statistics of its location. As shown by the positive correlation between balloon's positions and homing accuracy, the participants did not ignore the non-permanent landmark but most likely have learned the probability of the balloon's position. Interestingly, the relation between the prior of permanency and the relocation effect increased linearly. The learning is not driven by an on/off process, but instead in line with the probabilistic nature of the learning, participants learned to weight the use of the landmark according to the situation. As a matter of fact, none of the weights allocated to the landmark configuration equaled 0 . In conditions of a non-permanent prior, the weight is inversely related to the distance of the relocated landmark. The larger the distances of the relocated landmark were ( 4 and $6 \mathrm{~m}$ ), the lower were the weights allocated. This is the sign of an adaptive process. If we transfer this result to the time domain and go back to our introductory example in which you park your car, it is more likely that you use the church than a truck as a landmark. However, if you are only in the shop for a minute you may as well use the truck as a landmark because it is likely still there upon return. We interpret this result as a sign of a probabilistic learning.

Note that the discussion about the different variables is found in the General discussion section (homing performances, i.e., accuracy and precision, and the path travelled).

With the first experiment we confirmed our hypothesis: humans learn the feature of landmark's permanency in a probabilistic way. The goal of the second experiment is to test the influence of the statistics of the balloon repositioning on the behavior. Some objects are more permanent than others, what are the consequences of these priors on the navigational behavior? Loosely speaking, we test whether participants will use a tent and a car equally as landmarks, both being non-permanent but to different degrees, a car being less permanent than a tent.

\section{Experiment 2}


The first experiment showed that participants learned the feature of permanency in a probabilistic way. The second experiment was designed to test the influence of different priors on navigational performance. To that end, the position changes of the non-permanent landmark have been extended (compared to experiment 1) from an area with a radius of $9 \mathrm{~m}$ to $15 \mathrm{~m}$. Extending the area of repositioning should increase the possibility of detecting the feature of non-permanency for the participants.

\section{Method}

Participants. 32 participants ( 21 male, 11 female) took part in the first experiment aged between 18 and 47 ( $m=23.9 ; s d=5.2)$. The sample size was determined based on the Experiment 1 (Cohen's $d z=0.85$ ), aiming for a power of 0.95 with alpha $=0.05$ (two-tailed). All participants gave their written informed consent before participating and received course credit or money $(7 € / \mathrm{h})$. The experiment was approved by the ethics committee of Ulm University.

Material \& Procedure. Material and procedure were similar to experiment 1 (see Figure 2). The following paragraphs list only the differences between both experiments.

The statistics of the repositioning of the balloon was drawn from a normal distribution with a $15 \mathrm{~m}$ radius around its usual position (in experiment 1 , radius $=9 \mathrm{~m}$ ). In order to keep the triangle configuration, we prevented the balloon from being placed closer to the home position (on the $y$ axis). In consequence, the area was not round but flattened in the vicinity of the home position.

Experiment 2 tested only one relocation of $6 \mathrm{~m}$. This distance was chosen because it led to the biggest difference between both conditions in experiment 1 . The balloon was relocated $16 \mathrm{~m}$ from home along the $y$ axis (in experiment 1, four relocations were tested: $0,2,4$ and $6 \mathrm{~m}$ ).

The experiment was performed in only one session. All participants started with the permanent condition, then had a break of 7 minutes then performed the non-permanent condition. Both conditions started with 40 training trials followed by 80 mixed trials (40 testing trials and 40 training trials randomized). Each participant performed in total 240 trials ( $120 \times 2$ conditions). The experiment lasted around $2 \mathrm{~h} 30$ (see Figure 2 for details).

Results. Out of the 34 participants tested, two felt the effect of motion sickness preventing them to complete the entire experiment. All analysis is run on 32 participants. 
Homing accuracy. As shown in Figure 5 the training of landmark permanency has a significant effect on the accuracy of the homing responses. This pattern is confirmed by a one-way analysis of variance (ANOVA) performed on the mean accuracy of homing responses, with Permanency (permanent, non-permanent) as within-subjects factor. There was a main effect of Permanency, $F(1,63)=11.66, p=.001, \eta_{2} \mathrm{p}=0.16$, with higher value of accuracy for permanent than for non-permanent $(1.56 \mathrm{~m}$ vs $0.83 \mathrm{~m})$. The relocated landmark influenced more the permanent than the non-permanent conditions.

The weight allocated to the relocated landmark confirms the relocation effect. The participants' responses correspond to a weight of $0.42(1.5686 / 3.71)$ for the permanent condition, and a weight of $0.22(0.8335 / 3.71)$ for the non-permanent condition.

Finally, the correlation between balloon's positions and homing accuracy results in a positive correlation, $\sigma_{2}=0.40, p<.001$.

Homing precision. The training of landmark permanency has no significant effect on the precision of the homing responses. This pattern is confirmed by a one-way analysis of variance (ANOVA) performed on the mean precision of homing responses, with Permanency (permanent, non-permanent) as within-subjects factor. There was no main effect of Permanency, $F(1,63)=1.89, p=.17$ (permanent: 5.06 vs non-permanent: 6.23). Participants were equally constant in their responses in the permanent and the non-permanent conditions.

Trajectory's index. The path travelled by the participants is influenced by the training of landmark permanency (see Figure 5). This pattern is confirmed by a one-way analysis of variance (ANOVA) performed on the trajectory's index, with Permanency (permanent, nonpermanent) as within-subjects factor. There was a main effect of Permanency, $F(1,63)=5.38$, $p=.02, \eta_{2} \mathrm{p}=0.07$, with lower value for permanent than for non-permanent ( $0.60 \mathrm{vs} 0.67$ ).

[Figure 5 here] 


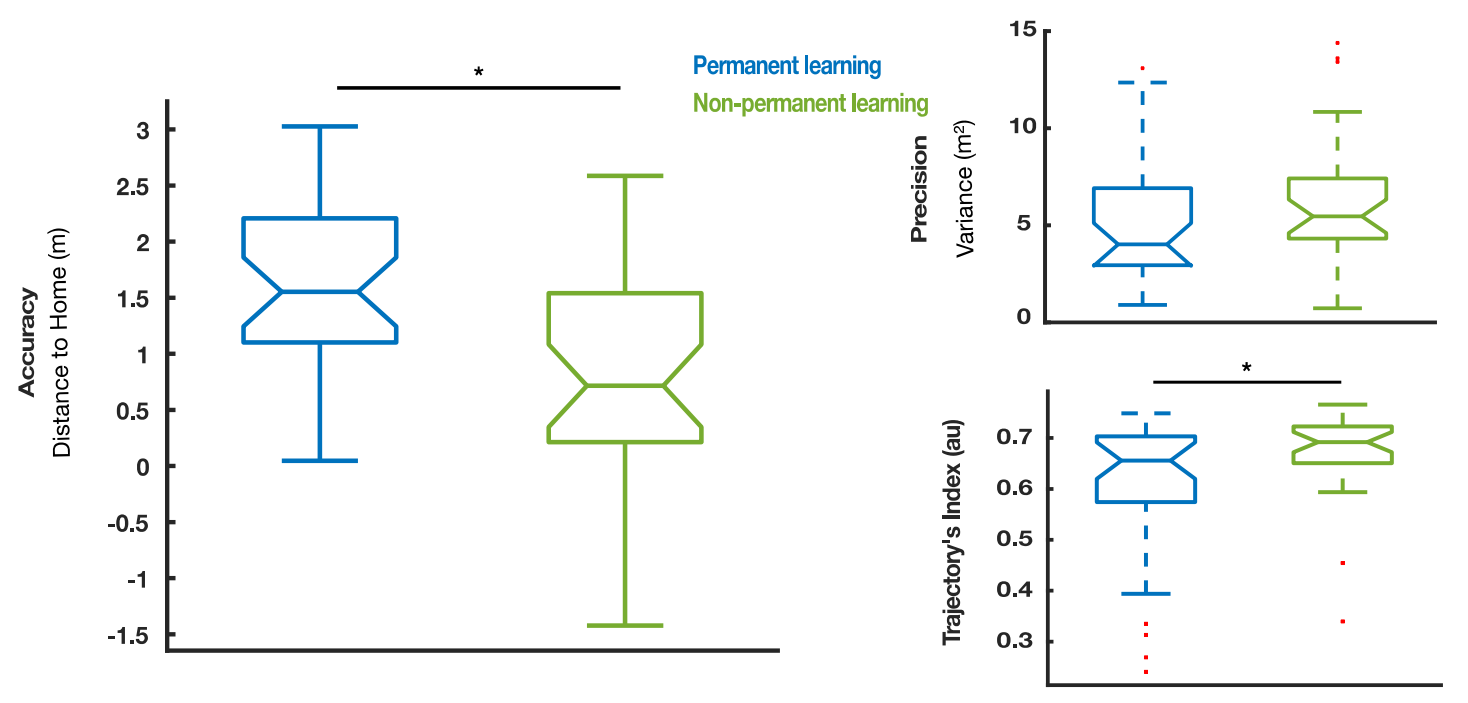

Figure 5. Results from Experiment 2. Boxplots of the accuracy, precision and goodness of trajectory for each learning condition: permanent in blue and non-permanent in green. Stars indicate significant differences between both conditions (permanent vs. non-permanent).

\section{Discussion.}

The navigational performance of the second experiment shows an effect of the prior of permanency, confirming that permanency is learned in a probabilistic fashion.

The goal of the second experiment was to test the influence of different priors on navigational performance. To evaluate the effect, we compared the navigational performance of experiment 1 with experiment 2 . The homing accuracy of the non-permanent condition (see Table 1) seems to confirm the effect of the manipulation (experiment 1 , relocation $6 \mathrm{~m}=1.27 \mathrm{~m}$ vs experiment $2=0.83 \mathrm{~m}$ ). Participants' responses in experiment 1 were more shifted toward the relocated balloon than participants' responses of experiment 2. Additionally, the difference between the permanent and the non-permanent conditions confirms the effect of the extended repositioning in experiment $1=-0.65(\mathrm{sd}=0.96)$ vs experiment $2=-0.73(\mathrm{sd}=1.02)$. The participants' responses in experiment 2 show on average a larger difference between both conditions compared to experiment 1. Last but not least, the weight allocated to the landmark's configuration of experiment 2 is lower than the weight of experiment 1 ( 0.22 versus 0.34 respectively). In conclusion, broadening the position change of the balloon decreased its influence as a landmark. Beyond confirming the results of the first experiment, the second experiment reinforces the hypothesis of a probabilistic learning. 
One could argue that, compared to experiment 2, in experiment 1 participants performed three times more trials ( 880 vs 240 trials). However, first the additional trials performed by participants of experiment 1 were equally distributed between the permanent and the nonpermanent conditions, participants did not learn more in one condition than in the other. Second, if the additional trials would have influenced the learning of permanency, it should have been in the direction of a larger effect of non-permanency on navigational behavior. The logic being that the participants of experiment 1 have more time to learn the feature of permanency than the participants of experiment 2. Therefore, if ever the number of trials had an effect on the performances, it should have been the other way around. In fact, the difference between the permanent and the non-permanent conditions is higher in the experiment 2 than in experiment 1 , so in absolute the effect is bigger in the experiment 2 than experiment 1.

With the first two experiments we revealed that humans learn the permanency feature of an object in a probabilistic fashion. The two last experiments will investigate the flexibility of the priors. Can participants update a prior? Once participants have learned that an object is nonpermanent, can they re-learn that it is permanent? In experiment 3 , the order of presentation has been reversed, investigating the symmetrical effect of the training.

\section{Experiment 3}

The third experiment was designed to test the symmetry of the training. The third experiment is similar to the second, but instead of starting with the permanent condition, participants started with the non-permanent condition. The goal of this experiment is to evaluate whether participants can update a previously trained prior of non-permanency to a prior of permanency for a same object.

\section{Method}

Participants. 17 participants ( 8 male, 9 female) took part in the first experiment aged between 19 and $40(m=24 ; s d=4.89)$. The sample size was determined based on experiment 2 (Cohen's $d z=0.85$ ), aiming for a power of 0.95 with alpha $=0.05$ (two-tailed). All participants gave their written informed consent before participating and received course credit or money $(7 € / \mathrm{h})$. The experiment was approved by the ethics committee of Ulm University. 
Material \& Procedure. Material and procedure were similar to experiment 2. Apart of the number of participants (Exp. $1 \mathrm{n}=33$ vs Exp. $3 \mathrm{n}=17$ ), the only difference between both experiments is the order of conditions. All participants started with the non-permanent condition, then had a break of 7 minutes and afterwards performed the permanent condition (see Figure 2).

Results. All analysis is run on 17 participants.

Homing accuracy. As can be seen in Figure 6, the training of landmark permanency has a significant effect on the accuracy of the homing responses. This pattern is confirmed by a oneway analysis of variance (ANOVA) performed on the mean accuracy of homing responses, with Permanency (permanent, non-permanent) as within-subjects factor. There was a main effect of Permanency, $F(1,33)=6.57, p=.015, \eta_{2} \mathrm{p}=0.17$, with higher value of accuracy for permanent than for non-permanent $(1.49 \mathrm{~m}$ vs $0.78 \mathrm{~m})$. The permanent condition is more influenced by the relocated landmark than the non-permanent condition.

The weight allocated to the relocated landmark confirms the relocation effect. The participants' responses correspond to a weight of $0.40(1.4915 / 3.71)$ for the permanent condition, and a weight of $0.21(0.7816 / 3.71)$ for the non-permanent condition.

Finally, the correlation between balloon's positions and homing accuracy results in a positive correlation, $\sigma_{2}=0.30, p<.001$.

Homing precision. As can be seen in Figure 6, the training of landmark permanency has a significant effect on the precision of the homing responses. This pattern is confirmed by a oneway analysis of variance (ANOVA) performed on the mean precision of homing responses, with Permanency (permanent, non-permanent) as within-subjects factor. There was a main effect of Permanency, $F(1,33)=5.23, p=.029, \eta_{2} \mathrm{p}=0.14$ (permanent: 4.46 vs non-permanent: 8.17). The participants were more constant in their responses for the permanent condition than for the non-permanent condition.

Trajectory's index. The path travelled by the participants is not influenced by the training of landmark permanency (see Figure 6). This pattern is confirmed by a one-way analysis of variance (ANOVA) performed on the trajectory's index, with Permanency (permanent, nonpermanent) as within-subjects factor. There was no main effect of Permanency, $F(1,33)=0.84$, $p=.36$. 
[Figure 6 here]
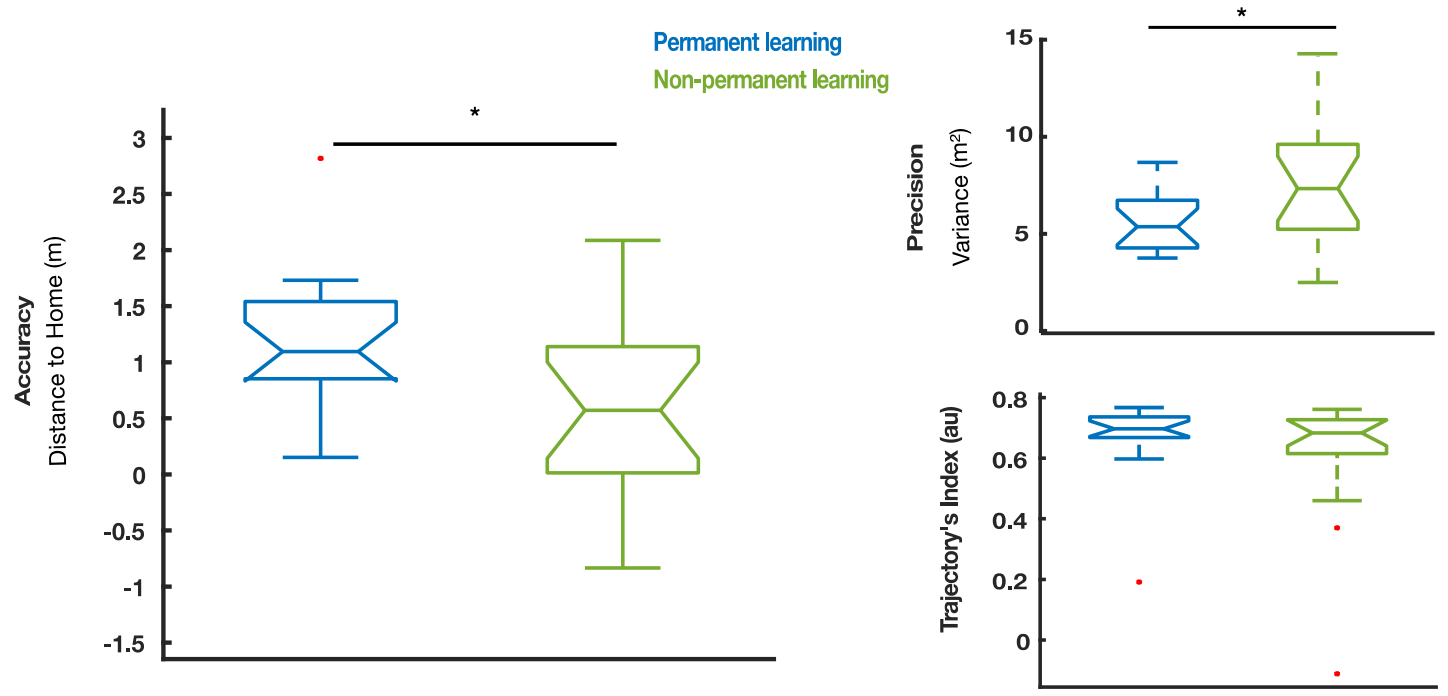

Figure 6. Results from Experiment 3. Boxplots of the accuracy, precision and goodness of trajectory for each learning condition: permanent in blue and non-permanent in green. Stars indicate significant differences between both conditions (permanent vs. non-permanent).

\section{Discussion.}

The third experiment confirms the results of the first two experiments, reinforcing our hypothesis: the participants' ability to locate home is related with the permanency of the object. Moreover, the feature of permanency is learned in a probabilistic fashion.

The goal of the third experiment was to investigate the flexibility of the prior of permanency, and particularly its capacity of updating from non-permanent to permanent. To that end, we compare the results of experiment 2 and 3. First, we can notice that the effect size of experiments 2 and 3 are similar $\left(\eta_{2} p=0.16\right.$ vs $\left.\eta_{2} p=0.17\right)$. Moreover, the weight allocated to the non-permanent conditions are also similar (0.22 and 0.21). The homing accuracy difference between the permanent and the non-permanent conditions are also similar for both experiments (experiment 2: mean $=-0.73, s d=1.02$; experiment 3: mean $=-0.71, s d=0.88$ ). Note that the number of participants of both experiments were different but that this should not influence the results. Altogether, the results indicated that the learning of permanency is symmetric. The prior can be updated in both directions, from permanent to non-permanent as well as from nonpermanent to permanent.

As for the fourth experiment, we wondered whether the ability of updating a prior can be extended to the object category. In experiment 3, we tested the capacity of updating a prior in 
a short-term period. The fourth experiment investigates whether humans are able to update a lifetime prior. As shown in Auger et al., (2012) study, we all learned that a house usually does not move but a hot-air balloon does. The objective of the fourth experiment is to test whether this knowledge can be updated. Can participants learn that a house is a non-permanent object? In experiment 4 , the hot-air balloon was replaced by the house and vice versa.

\section{Experiment 4}

The fourth experiment was designed to test the capacity of updating a lifetime prior. The objects, i.e., balloon, house and tree, of the three previous experiments have been chosen because of their properties of permanency (Auger et al., 2012). A balloon is rated less permanent than a house or a tree. The goal of the fourth experiment is to evaluate whether participants can learn that the house in our experiment is a non-permanent object. In line with the hypothesis of a probabilistic learning, i.e., repetitive experiences of a situation, we expect that participants will be able to update their priors and therefore consider the house as a nonpermanent object.

\section{Method}

Participants. 17 participants (11 male, 6 female) took part in the fourth experiment aged between 19 and 41 ( $m=23.59 ; s d=5.48)$. The sample size was determined based on experiment 2 (Cohen's $d z=0.85$ ), aiming for a power of 0.95 with alpha $=0.05$ (two-tailed). All participants gave their written informed consent before participating and received course credit or money $(7 € / \mathrm{h})$. The experiment was approved by the ethics committee of Ulm University.

Material \& Procedure. Material and procedure were similar to experiment 3 (see Figure 2) except for one aspect: the house represented the non-permanent object, while the permanent objects were represented by the balloon and the tree. The entire procedure remains the same as in experiment 3.

Results. All analysis is run on 17 participants. 
Homing accuracy. As can be seen in Figure 7, the training of landmark permanency has a significant effect on the accuracy of the homing responses. This pattern is confirmed by a oneway analysis of variance (ANOVA) performed on the mean accuracy of homing responses, with Permanency (permanent, non-permanent) as within-subjects factor. There was a main effect of Permanency, $F(1,33)=5.44, p=.026, \eta_{2} \mathrm{p}=0.15$, with higher value of accuracy for permanent than for non-permanent $(1.48 \mathrm{~m}$ vs $0.95 \mathrm{~m})$. The permanent condition is more influenced by the relocated landmark than the non-permanent condition.

The weight allocated to the relocated landmark confirms the relocation effect. The participants' responses correspond to a weight of $0.40(1.4797 / 3.71)$ for the permanent condition, and a weight of $0.26(0.9543 / 3.71)$ for the non-permanent condition.

Finally, the correlation between balloon's positions and homing accuracy results in a positive correlation, $\sigma_{2}=0.47, p<.001$.

Homing precision. As can be seen in Figure 7, the training of landmark permanency has no significant effect on the precision of the homing responses. This pattern is confirmed by a oneway analysis of variance (ANOVA) performed on the mean precision of homing responses, with Permanency (permanent, non-permanent) as within-subjects factor. There was no main effect of Permanency, $F(1,33)=0.52, p=.48$ (permanent: 5.25 vs non-permanent: 5.98). Participants' responses did not significantly differ in their ability to provide constant responses when comparing permanent and non-permanent conditions.

Trajectory's index. The path travelled by the participants is influenced by the training of landmark permanency (see Figure 7). This pattern is confirmed by a one-way analysis of variance (ANOVA) performed on the trajectory's index, with Permanency (permanent, nonpermanent) as within-subjects factor. There was a main effect of Permanency, $F(1,33)=4.05$, $p=.052, \eta_{2} \mathrm{p}=0.11$, with lower value for permanent than for non-permanent (0.65 vs 0.72).

[Figure 7 here] 


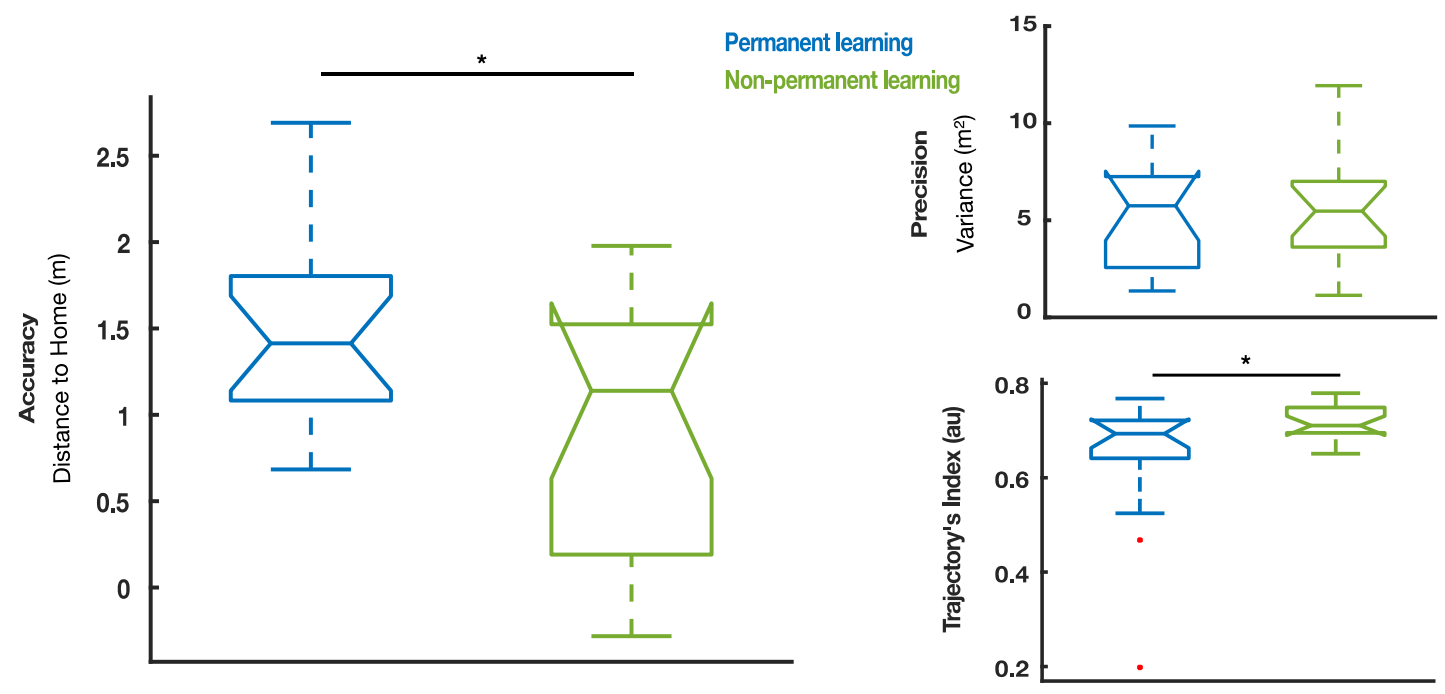

Figure 7. Results from Experiment 4. Boxplots of the accuracy, precision and goodness of trajectory for each learning condition: permanent in blue and non-permanent in green. Stars indicate significant differences between both conditions (permanent vs. non-permanent).

\section{Discussion.}

Here, we showed that participants were able to adapt their behavior to the requirements of the situation in updating the assumed prior of a house being a permanent landmark. The feature of permanency influenced the homing performances similarly whether the non-permanent object was a house or a balloon. The effect sizes of the experiments 2, 3 and 4 are similar (respectively $\left.\eta_{2} \mathrm{p}=0.16 ; \eta_{2} \mathrm{p}=0.17 ; \eta_{2} \mathrm{p}=0.15\right)$. Moreover, the weight allocated to the non-permanent conditions are also similar for the experiments 2,3 and 4 corresponding to $0.22,0.21$ and 0.26 respectively. Regarding the homing accuracy difference between permanent and nonpermanent, the differences were similar for experiment 2 and 3 (experiment 2: mean $=-0.73$, $s d=1.02$; experiment 3: mean $=-0.71, s d=0.88$ ), this last experiment 4 showed a smaller difference (experiment 4 : mean $=-0.52, s d=0.65$ ). We performed a one-way analysis of variance (ANOVA) on the differences between permanent vs non-permanent with the experiment as factor (experiment 3 vs experiment 4 ): $F(1,33)=0.48, p=.49$. There is no significant difference which confirms our hypothesis: participants had learned that a house can be a non-permanent landmark, as well as for a balloon. We showed here that participants updated their priors from permanency to non-permanency, simply via experiences. 
[Table 1 here]

Table 1

Summary table of the main results.

\begin{tabular}{|c|c|c|c|c|c|c|c|c|}
\hline & \multicolumn{4}{|c|}{ Permanent } & \multicolumn{4}{|c|}{ Non-Permanent } \\
\hline & Accuracy & Weight & Precision & Trajectory & Accuracy & Weight & Precision & Trajectory \\
\hline $\begin{array}{c}\text { Experiment } 1 \stackrel{\star}{\star} \\
4 \text { relocations }\end{array}$ & 1.91 & 0.52 & 3.28 & 0.65 & $1.27 *$ & 0.34 & $4.17 *$ & $0.68 *$ \\
\hline $\begin{array}{c}\text { Experiment } 2 \\
15 \text { m radius }\end{array}$ & 1.56 & 0.42 & 5.06 & 0.60 & $0.83 *$ & 0.22 & 6.23 & $0.67 *$ \\
\hline $\begin{array}{l}\text { Experiment } 3 \\
\quad \text { Non-permanent first }\end{array}$ & 1.49 & 0.40 & 4.46 & 0.67 & $0.78^{*}$ & 0.21 & $8.17 *$ & 0.61 \\
\hline Experiment 4 & & & & & & & & \\
\hline $\begin{array}{l}\text { House: non- } \\
\text { permanent }\end{array}$ & 1.48 & 0.40 & 5.25 & 0.65 & $0.95^{*}$ & 0.26 & 5.98 & $0.72 *$ \\
\hline
\end{tabular}

$\stackrel{\star}{\star}$ For experiment 1 , the results refer only to the $6 \mathrm{~m}$ relocation.

The Asterix $(*)$ stand for a significant difference between permanent and non-permanent conditions (see the results section for details).

\section{General Discussion}

In this series of experiments, we investigated how human learn a landmark's permanency to navigate. We hypothesized a probabilistic learning based on repetition of experiences. To that end, we studied the behavior of participants when exposed to two different landmark characteristics, permanent vs non-permanent. The results of all experiments showed that a permanent landmark influenced the navigational performances more than a non-permanent landmark, confirming that an object should be permanent to be used as a landmark. Interestingly, participants' behavior in the four experiments shows that experiences can modify a prior, which is a signature of a probabilistic learning. More appealing, based on this probabilistic learning participants have learned that a house can be a moving object. This last result revealed that priors are adaptable and can be modified according the requirements of the environment. 
In experiment 1 we showed that navigational performance is influenced by the prior of permanency. The repetitive experience of an object whose position changes reduces its use as a landmark to navigate. The weight allocated to the permanent landmark was higher than that allocated to the non-permanent, which is a signature of a probabilistic behavior.

In experiment 2, we showed that the manipulation of the statistics of the balloon, by extending the area of position changes, reduces its use as a landmark.

Experiments 3 and 4 showed that priors can be updated by experiences. In experiment 3 we found that, regardless of the precedence, a prior can be modified symmetrically. The last experiment revealed that a lifetime prior can be updated, leading the participants to regard a house as a moving object.

Our study is embedded in the Bayesian Decision Theory applied to navigational behavior. Some studies already investigated navigational behavior with the lens of the Bayesian theory (Cheng et al., 2007; Kiryakova et al., 2019; Nardini et al., 2008; Pfuhl et al., 2011). This theory provides a framework for a better understanding of navigational behavior. Our study investigated an important aspect of the navigation, the landmark-based strategy and particularly the feature of permanency of the landmarks. We showed here that this feature is not inherent to the object itself but is the consequence of learning. Altogether, the results of our four experiments confirm that the feature of permanency is a prior learned in a probabilistic fashion. For example, in the third experiment, the symmetry of updating the prior, from permanent to non-permanent and vice versa, is an additional evidence to support the hypothesis of a probabilistic learning. The behavior of participants showed that priors of a same object can be successively permanent and non-permanent. This ability to adapt can be useful in the reality. To go back to our example in the introduction in which you park your car, it is more likely that you use a church than a truck as a landmark. However, if you are only gone for a minute you may as well use the truck as a landmark, because it is likely still there upon return. One can argue that our experiment is set in a virtual environment where participants are more prone to accept alternative realities. Indeed, it will be interesting to confirm the effects in real life. However, the theory predicts the same pattern of results. As shown in the Adams et al., (2004) study, a lifetime experience of light coming from above can be modified through a $1 \mathrm{~h} 30$ training experience. We are certain that the repetitive experience of a house changing its position from trial to trial in real life will lead to the same result as a hot-air balloon, but it can be still appealing to test it.

Another interesting aspect of this study is the difference between the variables. So far, we have exclusively discussed the results in the light of the accuracy of the homing behavior, leaving 
out its precision. In general, the precision confirms the result found for the accuracy, i.e., influence of the prior of permanency on the navigation behavior. Two out of the four experiments reported that the homing performances are more precise, i.e., have lower variance, in the permanent conditions compared to the non-permanent conditions. Only two experiments reported significant differences. However, the results of the two others (experiment 2 and 4) are going in the same direction. These results are in line with a previous experiment which links the number of landmarks available to the precision of the homing performance (Jetzschke et al., 2017). The precision of the homing performance confirms the effect of the prior. The participants' responses were influenced more by the relocation effect in the permanent conditions and at the same time the responses were more precise, as participants used the three landmarks to locate the home position. However, in the non-permanent conditions, participants were using the two permanent landmarks while the non-permanent one only served as an indicator for the home positions location relative to the permanent landmarks. Consequently, the accuracy of the homing performance is less influenced by the relocation effect and parallelly the performances were less precise. Note that for all the experiments the non-permanent landmark, balloon or house, have never been ignored. The results showed that in the permanent conditions the landmarks influenced more the navigational performances than in the nonpermanent conditions. However, the non-permanent object has also been used because it always gave an indication of the home position's location relative to the permanent landmarks. Otherwise the responses would have been equally distributed between the real home position and at the exact opposite. A previous study has shown that responses are split in two distribution at two opposite positions when only two landmarks are available which don't provide any indication of direction (Jetzschke et al., 2017). To conclude on the status of the homing performances, precision and accuracy tell us the same story and support the conclusion of the influence of the permanency prior on the homing behavior.

Interestingly, the ability to locate home and the path travelled to home seemed to be triggered by different information. Indeed, the path used by the participants seemed to be more influenced by the order of presentation than by the landmark's permanency. For all experiments, the participants were going straighter to home during the second part of the experiment, which corresponds to the permanent conditions in experiment 1,2 and 4, and to the non-permanent condition in experiment 3 . Note that the difference between the two conditions in experiment 3 are not significant but the performances are reversed, the paths were straighter in the permanent condition than in the non-permanent one. Therefore, it seems that the trajectory of the path is an index of how well participants learn to navigate in the setup, going straighter at the end of 
the experiment than at the beginning. At first the participants might need time to learn the controls and to orientate themselves, but after some trials they surely have learned and choose straighter paths than at the beginning. It is interesting to note that homing performances and path's trajectory seem to measure different abilities. These results might suggest that finding home is a decision which happens at the very last moment, while the way to go back home seems more influenced by the ability to move physically in the environment. In that respect, the feature of permanency of an object facilitates the ability to locate home but not the ability to go back to the home place. However, as pointed out the results of experiment 3 are not statistically significant, it could suggest a slight effect of permanency on the path travelled to go home. This possibility will need further investigation.

In conclusion, we first confirmed that landmark's permanency is a crucial feature for navigation because it facilitates our ability to locate home. Second, we showed that learning this feature of permanency is accomplished in a probabilistic fashion and that priors can be updated by experiences highlighting the human capacity of adaptation to the environment. The next step would be to test the capacity of transferring the learned to other situations. An additional evidence that this probabilistic learning follows Bayesian principles would be to show that the prior of permanency, learned in a context of homing task navigation, is transferable to another type of task, since the capacity of transfer is a signature of the Bayesian principles (Kiryakova et al., 2019; Maloney \& Mamassian, 2009). To conclude, navigation is one of the most fundamental abilities in animals including humans. We showed here that humans are animals decidedly adaptable. 


\section{References}

Adams, W. J., Graf, E. W., \& Ernst, M. O. (2004). Experience can change the "light-fromabove" prior. Nature Neuroscience, 7(10), 1057-1058. https://doi.org/10.1038/nn1312

Auger, S. D., \& Maguire, E. A. (2013). Assessing the mechanism of response in the retrosplenial cortex of good and poor navigators. Cortex, 49(10), 2904-2913. https://doi.org/10.1016/j.cortex.2013.08.002

Auger, S. D., Mullally, S. L., \& Maguire, E. A. (2012). Retrosplenial Cortex Codes for Permanent Landmarks. PLoS ONE, 7(8), e43620. https://doi.org/10.1371/journal.pone.0043620

Auger, S. D., Zeidman, P., \& Maguire, E. A. (2017). Efficacy of navigation may be influenced by retrosplenial cortex-mediated learning of landmark stability. Neuropsychologia, 104, 102-112. https://doi.org/10.1016/j.neuropsychologia.2017.08.012

Bécu, M., Sheynikhovich, D., Tatur, G., Agathos, C. P., Bologna, L. L., Sahel, J.-A., \& Arleo, A. (2019). Age-related preference for geometric spatial cues during real-world navigation. Nature Human Behaviour. https://doi.org/10.1038/s41562-019-0718-z

Biegler, R., \& Morris, R. G. M. (1996). LANDMARK STABILITY: STUDIES

\section{EXPLORING WHETHER THE PERCEIVED STABILITY OF THE} ENVIRONMENT INFLUENCES SPATIAL REPRESENTATION. Journal of Experimental Biology, 199(1), 7.

Burgess, N., Spiers, H., \& Paleologou, E. (2004). Orientational manoeuvres in the dark: Dissociating allocentric and egocentric influences on spatial memory. Cognition, 94(2), 149-166. https://doi.org/10.1016/j.cognition.2004.01.001

Burnett, G. (2000). 'Turn right at the Traffic Lights': The Requirement for Landmarks in Vehicle Navigation Systems. Journal of Navigation, 53(August), 499-510. https://doi.org/10.1017/S0373463300001028 
Caduff, D., \& Timpf, S. (2008). On the assessment of landmark salience for human navigation. Cognitive Processing, 9(4), 249-267. https://doi.org/10.1007/s10339-0070199-2

Cheng, K., Shettleworth, S. J., Huttenlocher, J., \& Rieser, J. J. (2007). Bayesian integration of spatial information. Psychological Bulletin, 133(4), 625-637. https://doi.org/10.1037/0033-2909.133.4.625

Dyck, E., Zell, E., Kohsik, A., \& Grewe, P. (2012). OCTAVIS: An Easy-to-Use VR-System for Clinical Studies. 10.

Ernst, M. O., \& Bülthoff, H. H. (2004). Merging the senses into a robust percept. Trends in Cognitive Sciences, 8(4), 162-169. https://doi.org/10.1016/j.tics.2004.02.002

Etchamendy, N., \& Bohbot, V. D. (2007). Spontaneous navigational strategies and performance in the virtual town. Hippocampus, 17(8), 595-599. https://doi.org/10.1002/hipo.20303

Etienne, A. S., \& Jeffery, K. J. (2004). Path integration in mammals. Hippocampus, 14(2), 180-192. https://doi.org/10.1002/hipo.10173

Foo, P., Warren, W. H., Duchon, A., \& Tarr, M. J. (2005). Do Humans Integrate Routes Into a Cognitive Map? Map- Versus Landmark-Based Navigation of Novel Shortcuts. Journal of Experimental Psychology: Learning, Memory, and Cognition, 31(2), 195215. https://doi.org/10.1037/0278-7393.31.2.195

Geva-Sagiv, M., Las, L., Yovel, Y., \& Ulanovsky, N. (2015). Spatial cognition in bats and rats: From sensory acquisition to multiscale maps and navigation. Nature Reviews Neuroscience, 16(2), 94-108. https://doi.org/10.1038/nrn3888

IglÃ si, K., Zaoui, M., Berthoz, A., \& Rondi-Reig, L. (2009). Sequential egocentric strategy is acquired as early as allocentric strategy: Parallel acquisition of these two navigation strategies. Hippocampus, 19(12), 1199-1211. https://doi.org/10.1002/hipo.20595 
Jetzschke, S., Ernst, M. O., Froehlich, J., \& Boeddeker, N. (2017). Finding Home: Landmark Ambiguity in Human Navigation. Frontiers in Behavioral Neuroscience, 11. https://doi.org/10.3389/fnbeh.2017.00132

Kiryakova, R., Aston, S., Beierholm, U., \& Nardini, M. (2019). Bayesian transfer in a complex spatial localisation task [Preprint]. Neuroscience. https://doi.org/10.1101/716431

Knierim, J. J., Kudrimoti, H. S., \& McNaughton, B. L. (1998). Interactions Between Idiothetic Cues and External Landmarks in the Control of Place Cells and Head Direction Cells. Journal of Neurophysiology, 80(1), 425-446. https://doi.org/10.1152/jn.1998.80.1.425

Maloney, L. T., \& Mamassian, P. (2009). Bayesian decision theory as a model of human visual perception: Testing Bayesian transfer. Visual Neuroscience, 26(1), 147-155. https://doi.org/10.1017/S0952523808080905

Montello, D. R. (2005). Navigation. In P. Shah \& A. Miyake (Eds.), The Cambridge Handbook of Visuospatial Thinking (pp. 257-294). Cambridge University Press. https://doi.org/10.1017/CBO9780511610448.008

Nardini, M., Jones, P., Bedford, R., \& Braddick, O. (2008). Development of Cue Integration in Human Navigation. Current Biology, 18(9), 689-693. https://doi.org/10.1016/j.cub.2008.04.021

Parise, C. V., Knorre, K., \& Ernst, M. O. (2014). Natural auditory scene statistics shapes human spatial hearing. Proceedings of the National Academy of Sciences, 111(16), 6104. https://doi.org/10.1073/pnas.1322705111

Pfuhl, G., Tjelmeland, H., \& Biegler, R. (2011). Precision and Reliability in Animal Navigation. Bulletin of Mathematical Biology, 73(5), 951-977. https://doi.org/10.1007/s11538-010-9547-y 
Sorrows, M. E., \& Hirtle, S. C. (1999). The Nature of Landmarks for Real and Electronic Spaces. In C. Freksa \& D. M. Mark (Eds.), Spatial Information Theory. Cognitive and Computational Foundations of Geographic Information Science (Vol. 1661, pp. 3750). Springer Berlin Heidelberg. https://doi.org/10.1007/3-540-48384-5_3 